\title{
Polarization-selective optical nonlinearities in cold Rydberg atoms
}

\author{
Jin-Hui Wu, ${ }^{1}$ M. Artoni, ${ }^{2,3}$ and G. C. La Rocca ${ }^{4}$ \\ ${ }^{1}$ Center for Quantum Sciences, Northeast Normal University, Changchun 130117, China \\ ${ }^{2}$ European Laboratory for Nonlinear Spectroscopy (LENS), 50019 Firenze, Italy \\ ${ }^{3}$ Department of Engineering and Information Technology \& INO-CNR, Brescia University, 25133 Brescia, Italy \\ ${ }^{4}$ Scuola Normale Superiore and CNISM, 56126 Pisa, Italy \\ (Received 26 July 2015; published 3 December 2015)
}

\begin{abstract}
We study the interaction between a probe and a trigger weak fields in a sample of cold rubidium atoms in the presence of a coupling and a dressing strong fields. Dipole Rydberg blockade may occur and can be set to depend on the probe and trigger polarizations giving rise to diverse regimes of electromagnetically induced transparency (EIT) with a concomitant small probe and trigger absorption and dispersion. This is shown to be relevant to the implementation of polarization conditional probe and trigger cross nonlinearities in cold Rydberg atoms.
\end{abstract}

DOI: 10.1103/PhysRevA.92.063805

PACS number(s): 42.50.Gy, 03.67.Lx, 32.80.Ee, 42.65.-k

\section{INTRODUCTION}

Photon-photon interactions enabled by nonlinear optical mechanisms play an important role in quantum information processing. Photons, in fact, are ideal carriers of quantum information as they can propagate at the speed of light and are generally not affected by the environment [1]. Large cross-phase nonlinearities between two photons, in particular, are crucial in many quantum information applications. One of the preferred and widely explored schemes to enhance cross-phase nonlinearities is based on Kerr-EIT interactions between a probe field and a signal field [2-5]. A major drawback, in this case, is the mismatch of group velocities which limits the probe and signal interaction time, placing an upper limit on the order of a small fraction of a radian to the resulting cross-phase shift [6]. Large intensities are required instead to reach appreciable nonlinear modulations using Kerr-EIT-like interactions [7]. To overcome this limit, matched double slow-light pulses with two atomic species [8] have been proposed as well as various other variant schemes including the tripod [9-11], N-tripod [12,13], and M [14,15] configurations. Enhanced cross-phase modulations have been implemented with a double EIT experiment in the tripod configuration of ${ }^{87} \mathrm{Rb}$ atoms [3] and a double slow-light experiment in the $\mathrm{M}$ configuration of ${ }^{133} \mathrm{Cs}$ atoms [4].

Cold Rydberg atoms, on the other hand, are now attracting great attention $[16,17]$. The unique combination of their strong dipole-dipole interactions and long radiative lifetimes can, in fact, be exploited to realize robust light-atom quantum interfaces [18-20] for quantum information processing purposes. Rydberg atoms may exhibit, in particular, a special cooperative nonlinearity [21] known as Rydberg blockade, whereby the presence of an atom excited to a Rydberg state will shift out of resonance the corresponding Rydberg state of all nearby atoms prohibiting the simultaneous Rydberg excitation of two or more atoms within a given volume (corresponding to a so-called superatom) determined by their dipole-dipole interactions. Strong photonic interactions in the presence of a Rydberg blockade have been shown to occur in samples of cold Rydberg atoms when driven into a EIT regime [22,23]. This has been restrained, however, to considerations of absorptive and dispersive photon-photon interactions of a single light field.
Extensions to conditional nonlinear interactions between two light fields are usually not straightforward [24-27].

The paper's main motivation is the discussion of an efficient scheme for achieving large conditional nonlinear interactions between two weak optical fields with a concomitant small absorption and dispersion. We show that such conditional interactions can be achieved in samples of cold rubidium atoms through the polarization-selective excitation of a high-lying Rydberg state coupled by a probe field and a trigger field in different EIT regimes. Specifically this is implemented under a symmetric EIT driving either through the inverted- $\Psi(P)$ configuration of Fig. 1(a) or through the inverted-Y (Y) configurations of Figs. 1(b) and 1(c). First, this extends typical cooperative nonlinearities observed in cold Rydberg atoms, whose nonlinear response depends on the incident field intensity at a fixed polarization [28-30], to polarization-conditional cooperative nonlinearities. Second, different cooperative nonlinearities arising from specific probe and trigger polarization choices, through the selection of specific Rydberg excitation paths, give rise to different optical cross nonlinearities between the probe and trigger fields. Polarization-conditional interactions between the two weak fields enable us to devise polarization-encoded cross nonlinearities exhibiting a large cross-phase modulation effect with little losses. Third, our polarization-conditional scheme takes place within the same ${ }^{87} \mathrm{Rb}$ superatom, at variance with what occurs, e.g., in cross-Kerr-like [31], resonant absorbing [24], transversely separated [25], and site addressable [26] Rydberg nonlinearities. Last and at variance with a simple cross-Kerr gate that depends on the intensity of a control field (scalar gate) [32-34], our polarization-selective optical nonlinearities scheme may be relevant to a Rydberg polarization gate. The latter is instead a vector gate, with two or more photon states simultaneously present, able to implement vector-state manipulations needed for quantum information processing.

We describe the evolution of internal variables for cold rubidium atoms through a standard set of Liouville equations briefly illustrated in Sec. II, while the atomic responses to both probe and trigger fields in one inverted $-\Psi$ and in two inverted$Y$ configurations are discussed, respectively, in Sec. III A and Secs. III B and IIIC. The Rydberg blockade effect is discussed here by treating all atoms through a superatom (SA) model suitably including the two-photon correlation [30,35]. 


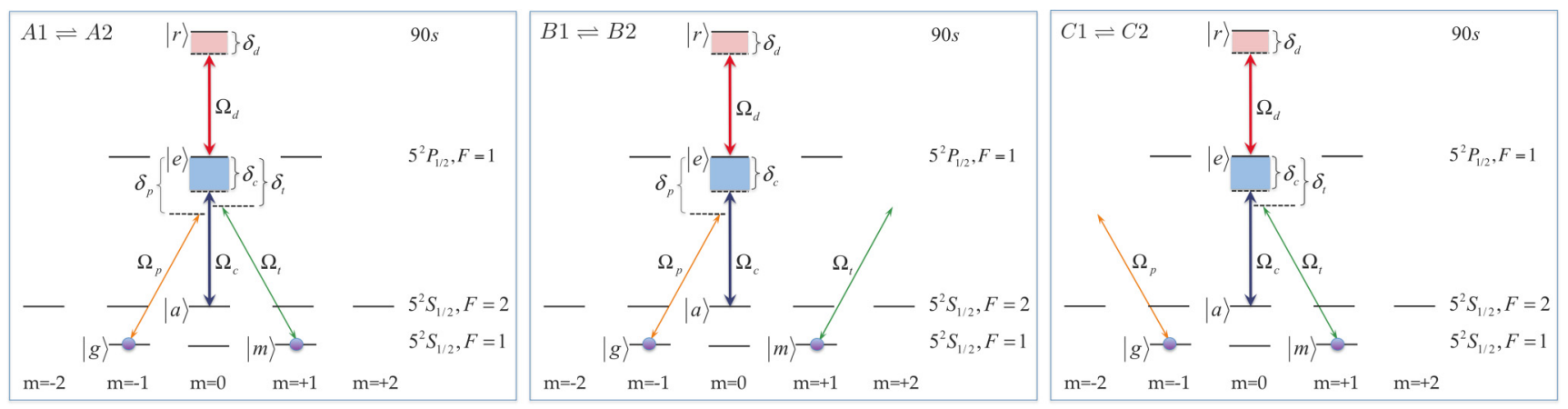

FIG. 1. (Color online) Level diagrams of cold ${ }^{87} \mathrm{Rb}$ atoms driven into the inverted- $\Psi(A 1)$ and the inverted-Y $(B 1, C 1)$ configurations. All atoms are equally distributed between the two ground levels $|g\rangle$ and $|m\rangle$ and transitions to the excited level $|e\rangle$ are induced by weak probe $\omega_{p}$ $(A, B)$ and trigger $\omega_{t}(A, C)$ fields, respectively. The excited level $|e\rangle$ is further connected to ground level $|a\rangle$ and Rydberg level $|r\rangle$ by strong coupling $\left(\omega_{c}\right)$ and dressing $\left(\omega_{d}\right)$ fields, respectively. The excitation of level $|r\rangle$ depends on the specific (circular) probe and trigger polarizations and so is the Rydberg blockade effect (see text). Atoms inside a SA containing one Rydberg excitation are driven into the tripod ( $A 2)$ or lambda $(B 2, C 2)$ configuration since strong dipole-dipole interactions now move level $|r\rangle$ far off resonance. Such polarization-selective dipole-dipole interactions give rise to two correlated EIT regimes, namely, "inverted- $\Psi \rightleftharpoons \operatorname{tripod}$ " $(A 1 \rightleftharpoons A 2)$ regime and "inverted-Y $\rightleftharpoons$ lambda" $(B 1 \rightleftharpoons B 2$ and $C 1 \rightleftharpoons C 2$ ) regime.

A detailed account of SA Rydberg populations resulting from the three different polarization-dependent excitation paths of Fig. 1 is given in Secs. IV A and IV B. Depending on whether the high Rydberg state is excited (dipole blockade) and how the polarization-excitation path is selected, different cooperative Rydberg nonlinearities are observed. These will give rise in turn to different cooperative dispersion and absorption effects, which we study in Sec. V for a pair of monochromatic probe and trigger fields under the three polarization configurations of Fig. 1. Concluding remarks about this paper's main results and their implications for polarization-encoded phase-gate applications are given in Sec. VI.

\section{THE BASIC MODEL}

Our aim is to exploit the Rydberg blockade effect to devise a polarization-selective scheme leading to large cross nonlinearities between two weak optical fields, say a probe and a trigger, yet minimizing losses. We further seek a driving configuration that is symmetric as possible with respect to the probe and trigger transitions so as to avoid any group velocity mismatch between them as they simultaneously propagate through a sample of cold atoms.

A symmetric inverted-Y level configuration [36] would actually allow us to couple the probe and trigger fields, respectively, to a pair of transitions from two different ground states to a common excited state which is, in turn, coupled to a higher Rydberg state via a strong dressing field in a ladderlike EIT scheme (see Fig. 1). The distinct selection rules of the two lower transitions provide the required dependence on probe and trigger polarizations. However, when Rydberg blockade is turned on detuning the Rydberg state out of resonance, this inverted-Y configuration would become a highly absorbing one. Yet, by adding a strong coupling field resonant with the transition from a third ground state to the intermediate excited state, so as to realize a symmetric inverted- $\Psi$ level configuration [Fig. 1(a)], we can well prevent absorption. Even when the Rydberg state is shifted out of resonance making the dressing field immaterial, this coupling field allows for a nearly lossless propagation of the probe and trigger fields in a lambdalike EIT scheme.

Probe and trigger will then exhibit in general different absorption and dispersion properties depending on their polarizations. The inverted $-\Psi$ configuration, in particular, appears to be a natural choice to achieve the above tasks of polarization selectivity and symmetric driving accompanied by small losses. This EIT scheme, where probe and trigger are treated on equal footing, is also relevant to cross-phase modulation applications.

\section{A. Symmetric inverted- $\Psi$ configuration}

We specifically consider an elongated sample of $N$ cold ${ }^{87} \mathrm{Rb}$ atoms located at fixed positions and illuminated by two strong laser fields of amplitudes $\mathbf{E}_{c}$ and $\mathbf{E}_{d}$ and two weak laser fields of amplitudes $\mathbf{E}_{p}$ and $\mathbf{E}_{t}$. The coupling $\left(\omega_{c}\right)$ and dressing $\left(\omega_{d}\right)$ fields are assumed to have fixed linear polarizations, while the probe $\left(\omega_{p}\right)$ and trigger $\left(\omega_{t}\right)$ fields may change from left to right circular polarizations or vice versa as shown in Figs. 1(a)-1(c).

The inverted- $\Psi$ configuration (case $A 1$ ), with transitions $|g\rangle \leftrightarrow|e\rangle,|m\rangle \leftrightarrow|e\rangle,|a\rangle \leftrightarrow|e\rangle$, and $|e\rangle \leftrightarrow|r\rangle$, is attained with the choice of "allowed" circular polarizations, namely, a right-circular probe polarization $\left(\sigma_{p}^{+}\right)$and a left-circular trigger polarization $\left(\sigma_{t}^{-}\right)$. We further assume that (i) only levels $|g\rangle$ and $|m\rangle$ are initially populated and (ii) both probe and trigger fields are so weak that levels $|a\rangle,|e\rangle$, and $|r\rangle$ are approximately empty. The resulting frequency detunings (Rabi frequencies) are defined as $\delta_{p}=\omega_{p}-\omega_{e g}\left(\Omega_{p}=\mathbf{E}_{p} \mathbf{d}_{g e} / 2 \hbar\right), \delta_{t}=\omega_{t}-$ $\omega_{e m}\left(\Omega_{t}=\mathbf{E}_{t} \mathbf{d}_{m e} / 2 \hbar\right), \delta_{c}=\omega_{c}-\omega_{e a}\left(\Omega_{c}=\mathbf{E}_{c} \mathbf{d}_{a e} / 2 \hbar\right)$, and $\delta_{d}=\omega_{d}-\omega_{r e}\left(\Omega_{d}=\mathbf{E}_{d} \mathbf{d}_{e r} / 2 \hbar\right)$, with $\omega_{i j}\left(\mathbf{d}_{i j}\right)$ being the transition frequencies (dipole moments).

Adopting the rotating-wave and electric-dipole approximations, we first write down the interaction Hamiltonian for an ensemble of $N$ cold ${ }^{87} \mathrm{Rb}$ atoms in the symmetric inverted- $\Psi$ $(P)$ configuration (The superscript $P$ in the expressions for susceptibilities and populations refers to the configuration $A 1$, while the superscript $Y$ refers to the configuration $B 1$ and 
$C$ 1. Similarly, the superscript $T$ refers to the configuration $A 2$ while $L$ refers to the configurations $B 2$ and $C 2$.) as

$$
\begin{aligned}
H_{I}= & -\hbar \sum_{k=1}^{N}\left[\delta_{p} \sigma_{e e}^{P k}+\Delta_{m} \sigma_{m m}^{P k}+\Delta_{a} \sigma_{a a}^{P k}+\Delta_{r} \sigma_{r r}^{P k}\right] \\
& -\hbar \sum_{k=1}^{N}\left[\Omega_{p} \sigma_{e g}^{P k}+\Omega_{t} \sigma_{e m}^{P k}+\Omega_{c} \sigma_{e a}^{P k}+\Omega_{d} \sigma_{r e}^{P k}\right. \\
& + \text { H.c. }],
\end{aligned}
$$

with $\Delta_{m}=\left(\delta_{p}-\delta_{t}\right), \Delta_{a}=\left(\delta_{p}-\delta_{c}\right)$, and $\Delta_{r}=\left(\delta_{p}+\delta_{d}\right)$ defined as two-photon detunings. After a standard averaging of local atomic operators

$$
\frac{1}{\delta n} \sum_{k=1}^{\delta n} \sigma_{i j}^{P k} \rightarrow \sigma_{i j}^{P}(z)
$$

in a microvolume $\delta V$ containing $\delta n$ atoms centered at $z$, we obtain the following Liouville equations:

$$
\begin{aligned}
\partial_{t} \sigma_{g e}^{P} & =-\gamma_{g e}^{\prime} \sigma_{g e}^{P}+i \Omega_{d}^{*} \sigma_{g r}^{P}+i \Omega_{c} \sigma_{g a}^{P}+i \Omega_{t} \sigma_{g m}^{P}+i \Omega_{p} \sigma_{g g}^{P}, \\
\partial_{t} \sigma_{g a}^{P} & =-\gamma_{g a}^{\prime} \sigma_{g a}^{P}+i \Omega_{c}^{*} \sigma_{g e}^{P}, \\
\partial_{t} \sigma_{g r}^{P} & =-\gamma_{g r}^{\prime} \sigma_{g r}^{P}+i \Omega_{d} \sigma_{g e}^{P}, \\
\partial_{t} \sigma_{m e}^{P} & =-\gamma_{m e}^{\prime} \sigma_{m e}^{P}+i \Omega_{d}^{*} \sigma_{m r}^{P}+i \Omega_{c} \sigma_{m a}^{P}+i \Omega_{p} \sigma_{m g}^{P}+i \Omega_{t} \sigma_{m m}^{P}, \\
\partial_{t} \sigma_{m a}^{P} & =-\gamma_{m a}^{\prime} \sigma_{m a}^{P}+i \Omega_{c}^{*} \sigma_{m e}^{P}, \\
\partial_{t} \sigma_{m r}^{P} & =-\gamma_{m r}^{\prime} \sigma_{m r}^{P}+i \Omega_{d} \sigma_{m e}^{P}, \\
\partial_{t} \sigma_{g m}^{P} & =-\gamma_{g m}^{\prime} \sigma_{g m}^{P}+i \Omega_{t}^{\dagger} \sigma_{g e}^{P}-i \Omega_{p} \sigma_{e m}^{P} .
\end{aligned}
$$

The remaining coherences and populations $\sigma_{e a}^{P}, \sigma_{e r}^{P}, \sigma_{a r}^{P}, \sigma_{e e}^{P}$, $\sigma_{a a}^{P}$, and $\sigma_{r r}^{P}$ are negligible in the limit of weak probe and trigger fields. We have also phenomenologically introduced the complex dephasing rates $\gamma_{g e}^{\prime}=\gamma_{g e}-i \delta_{p}, \gamma_{g a}^{\prime}=\gamma_{g a}-$ $i \Delta_{a}, \gamma_{g r}^{\prime}=\gamma_{g r}-i \Delta_{r}, \gamma_{g m}^{\prime}=\gamma_{g m}-i \Delta_{m}, \gamma_{m e}^{\prime}=\gamma_{m e}-i \delta_{t}$, $\gamma_{m a}^{\prime}=\gamma_{m a}-i \Delta_{a}^{\prime}$, and $\gamma_{m r}^{\prime}=\gamma_{m r}-i \Delta_{r}^{\prime}$ in terms of the real dephasing rates $\gamma_{g e}, \gamma_{g a}, \gamma_{g r}, \gamma_{g m}, \gamma_{m e}, \gamma_{m a}$, and $\gamma_{m r}$ as well as the two-photon detunings $\Delta_{a}^{\prime}=\delta_{t}-\delta_{c}$ and $\Delta_{r}^{\prime}=\delta_{t}+\delta_{d}$.

Notice that the inverted- $\Psi$ configuration, associated with the choice of allowed circular polarizations and the absence of Rydberg blockade, i.e., the case (A1) in Fig. 1, is described by fully symmetric equations. By switching one or the other circular polarization from allowed to forbidden, the full $\Psi$ configuration reduces to the asymmetric inverted-Y configuration $(B 1)$ or $(C 1)$ of Fig. 1. By switching both circular polarizations from allowed to forbidden we end up with a trivially symmetric and vacuumlike situation being both probe and trigger decoupled. On the other hand, when Rydberg blockade is turned on and level $|r\rangle$ is pushed out of resonance, the full inverted- $\Psi$ configuration $(A 1)$ reduces to the symmetric tripod configuration $(A 2)$ while the inverted- $Y$ configuration $(B 1)$ reduces to the lambda configuration $(B 2)$ and so is for the reduction $(C 1) \rightarrow(C 2)$.

\section{B. Reduced configurations}

The polarization selectivity in Fig. 1 stems from the selection rules of the $|g\rangle \leftrightarrow|e\rangle$ and $|m\rangle \leftrightarrow|e\rangle$ transitions. In particular, the trigger field may be decoupled from the $|m\rangle \leftrightarrow$ $|e\rangle$ transition due to the forbidden circular polarization $\left(\sigma_{t}^{+}\right)$ which results into an inverted- $Y$ configuration encompassing levels $|g\rangle,|a\rangle,|e\rangle$, and $|r\rangle$ (B1). Similarly, in the other
inverted-Y configuration with levels $|m\rangle,|a\rangle,|e\rangle$, and $|r\rangle(C 1)$ the probe field is decoupled from the $|g\rangle \leftrightarrow|e\rangle$ transition due to a forbidden circular polarization $\left(\sigma_{p}^{-}\right)$. It is easy to attain the Liouville equations for the off-diagonal elements $\sigma_{i j}^{Y}$ of an inverted-Y configuration ( $B 1$ or $C 1$ ) by setting, respectively, $\Omega_{t}=0$ or $\Omega_{p}=0$ in Eqs. (3).

We further stress, in a mean-field sense, that one atom excited to a Rydberg level $|r\rangle$ shifts the same Rydberg level of all surrounding atoms due to strong dipole-dipole interactions (Rydberg blockade). Precisely, the excitation to $|r\rangle$ of atoms within a blockade sphere of radius $R_{b}$ around the Rydberg excited atom is then strictly forbidden owing to a very large resonance shift. Consequently, within this blockade sphere, the optical responses to probe and trigger fields are no longer described by the inverted- $\Psi$ (A1) or inverted-Y $(B 1, C 1)$ EIT configurations, but rather by the basic tripod $(A 2)$ or lambda $(B 2, C 2)$ EIT configurations (see Fig. 1). Relevant Liouville equations for off-diagonal elements $\sigma_{i j}^{T}$ and $\sigma_{i j}^{L}$ in the tripod and lambda configurations can be easily attained by further setting $\Omega_{d}=0$ in the equations for $\sigma_{i j}^{P}$ and $\sigma_{i j}^{Y}$ of the inverted- $\Psi$ and inverted-Y configurations, respectively. A detailed discussion of how such polarization-selective dipoledipole interactions give rise to cooperative nonlinearities in correlated EIT regimes-inverted- $\Psi \rightleftharpoons \operatorname{tripod}(A 1 \rightleftharpoons A 2)$ and inverted-Y $\rightleftharpoons$ lambda $(B 1 \rightleftharpoons B 2$ and $C 1 \rightleftharpoons C 2)$-depending on the SA Rydberg populations will be provided in Sec. V.

\section{ATOMIC SUSCEPTIBILITIES}

We discuss in this section the probe and trigger susceptibilities for all six EIT configurations in Fig. 1. These can be obtained from Eqs. (3) via a straightforward, albeit lengthy, calculation of relevant steady-state probe $\left(\sigma_{g e}\right)$ and trigger $\left(\sigma_{m e}\right)$ atomic coherences, starting from the inverted- $\Psi(P)$ configuration $(A 1)$.

\section{A. Polarizations $\left\{\sigma_{p}^{+}, \sigma_{t}^{-}\right\}$}

For this choice of probe and trigger polarizations and for intensities such that $\left|\Omega_{p}\right|^{2} \sigma_{g g}=\left|\Omega_{t}\right|^{2} \sigma_{m m}$, the probe and trigger susceptibilities are

$$
\begin{aligned}
\chi_{p}^{P} & =\frac{N_{g} \sigma_{g e}^{P}}{\Omega_{p}} \\
& =N_{g} \frac{i \gamma_{g r}^{\prime} \gamma_{g a}^{\prime} \gamma_{g m}^{\prime} A_{m}^{*} \sigma_{g g}}{\gamma_{g m}^{\prime} A_{g} A_{m}^{*}+\gamma_{m r}^{\prime *} \gamma_{m a}^{\prime *} A_{g}\left|\Omega_{p}\right|^{2}+\gamma_{g r}^{\prime} \gamma_{g a}^{\prime} A_{m}^{*}\left|\Omega_{t}\right|^{2}}, \\
\chi_{t}^{P} & =\frac{N_{m} \sigma_{g m}^{P}}{\Omega_{t}} \\
& =N_{m} \frac{i \gamma_{m r}^{\prime} \gamma_{m a}^{\prime} \gamma_{m g}^{\prime} A_{g}^{*} \sigma_{m m}}{\gamma_{m g}^{\prime} A_{m} A_{g}^{*}+\gamma_{g r}^{\prime *} \gamma_{g a}^{\prime *} A_{m}\left|\Omega_{t}\right|^{2}+\gamma_{m r}^{\prime} \gamma_{m a}^{\prime} A_{g}^{*}\left|\Omega_{p}\right|^{2}},
\end{aligned}
$$

where $\sigma_{g e}^{P}$ and $\sigma_{m e}^{P}$ are steady-state solutions of Eqs. (3). We denote here by $N_{g}=N_{0} d_{e g}^{2} / 2 \varepsilon_{0} \hbar, N_{m}=N_{0} d_{e m}^{2} / 2 \varepsilon_{0} \hbar$, and $N_{0}$ the average volume density of a finite atomic sample of length $L$. We also define $A_{g}=\gamma_{g r}^{\prime} B_{g}+\gamma_{g a}^{\prime}\left|\Omega_{d}\right|^{2}$ and $B_{g}=\gamma_{g e}^{\prime} \gamma_{g a}^{\prime}+\left|\Omega_{c}\right|^{2}$ as well as $A_{m}=\gamma_{m r}^{\prime} B_{m}+\gamma_{m a}^{\prime}\left|\Omega_{d}\right|^{2}$ and $B_{m}=\gamma_{m e}^{\prime} \gamma_{m a}^{\prime}+\left|\Omega_{c}\right|^{2}$.

The inverted $-\Psi$ configuration $(A 1)$ can be seen as made of two adjacent inverted-Y configurations, one involving the 
probe field $(B 1)$ and the other involving the trigger field $(C 1)$, and both sharing the driving and coupling fields $\Omega_{d}$ and $\Omega_{c}$. Each inverted-Y configuration has, in turn, double dark states [36], whereby the probe field, for instance, exhibits double EIT $[3,37,38]$ leading to different transparency windows centered around $\delta_{p}=\delta_{c}$ and $\delta_{p}=-\delta_{d}$ with almost identical probe responses at both window centers. The two windows become centered at the same position when the dark states become degenerate, namely when $\delta_{c} \rightarrow-\delta_{d}$. Clearly the same holds unchanged for the trigger response.

The inverted- $\Psi$ configuration (A1), owing to its intrinsic symmetric driving structure, further allows for the tuning of these two pairs of dark states and, in particular, for their matching. This takes place when $\Omega_{p}=\Omega_{t}$ and $\delta_{p}=\delta_{t}$ and gives rise to similar absorptive and dispersive properties, hence similar group velocities, for both probe and trigger fields. Note, in fact, that under such a symmetric EIT driving the two expressions for $\chi_{p}^{P}$ and $\chi_{t}^{P}$ turn into each another upon the interchange $\{g \leftrightarrow m, p \leftrightarrow t\}$ [39]. Matching, in addition, yields almost identical probe responses in both inverted- $\Psi$ $(A 1)$ and inverted-Y $(B 1)$ configurations. Likewise, for a trigger field driving the $(A 1)$ and $(C 1)$ configurations.

Owing to dipole blockade, level $|r\rangle$ may be decoupled, which reduces the inverted- $\Psi$ configuration $(A 1)$ to a tripod configuration $(A 2)$ whose susceptibilities can easily be computed from Eqs. (3) by setting $\Omega_{d}=0$, i.e.,

$$
\begin{aligned}
\chi_{p}^{T} & =N_{g} \frac{i \gamma_{g a}^{\prime} \gamma_{g m}^{\prime} B_{m}^{*} \sigma_{g g}}{\left(\gamma_{g m}^{\prime} B_{g}+\gamma_{g a}^{\prime}\left|\Omega_{t}\right|^{2}\right) B_{m}^{*}+\gamma_{m a}^{\prime *}\left|\Omega_{p}\right|^{2} B_{g}}, \\
\chi_{t}^{T} & =N_{m} \frac{i \gamma_{m a}^{\prime} \gamma_{m g}^{\prime} B_{g}^{*} \sigma_{m m}}{\left(\gamma_{m g}^{\prime} B_{m}+\gamma_{m a}^{\prime}\left|\Omega_{p}\right|^{2}\right) B_{g}^{*}+\gamma_{g a}^{\prime *}\left|\Omega_{t}\right|^{2} B_{m}} .
\end{aligned}
$$

\section{B. Polarizations $\left\{\sigma_{p}^{+}, \sigma_{t}^{+}\right\}$}

The probe susceptibility associated with this polarizations choice corresponds to an inverted-Y configuration $(B 1)$ obtained by setting $\Omega_{t}=0$ in Eqs. (3),

$$
\chi_{p}^{Y}=N_{g} \frac{i \gamma_{g r}^{\prime} \gamma_{g a}^{\prime} \sigma_{g g}}{A_{g}},
$$

which reduces, in the presence of dipole blockade $\left(\Omega_{t}=\Omega_{d}=\right.$ $0)$, to that of a lambda configuration (B2),

$$
\chi_{p}^{L}=N_{g} \frac{i \gamma_{g a}^{\prime} \sigma_{g g}}{\gamma_{g e}^{\prime} \gamma_{g a}^{\prime}+\left|\Omega_{c}\right|^{2}} .
$$

In this case the trigger susceptibility vanishes as in vacuum $\left(\chi_{t}^{v}=0\right)$.

\section{Polarizations $\left\{\sigma_{p}^{-}, \sigma_{t}^{-}\right\}$}

This is instead the reverse situation in which the probe field has a vanishing susceptibility $\left(\chi_{p}^{v}=0\right)$, while the trigger susceptibility corresponds to that of an inverted- $Y$ configuration $(C 1)$,

$$
\chi_{t}^{Y}=N_{m} \frac{i \gamma_{m r}^{\prime} \gamma_{m a}^{\prime} \sigma_{m m}}{B},
$$

or to that of a lambda configuration $(C 2)$,

$$
\chi_{t}^{L}=N_{m} \frac{i \gamma_{m a}^{\prime} \sigma_{m m}}{\gamma_{m e}^{\prime} \gamma_{m a}^{\prime}+\left|\Omega_{c}\right|^{2}} .
$$
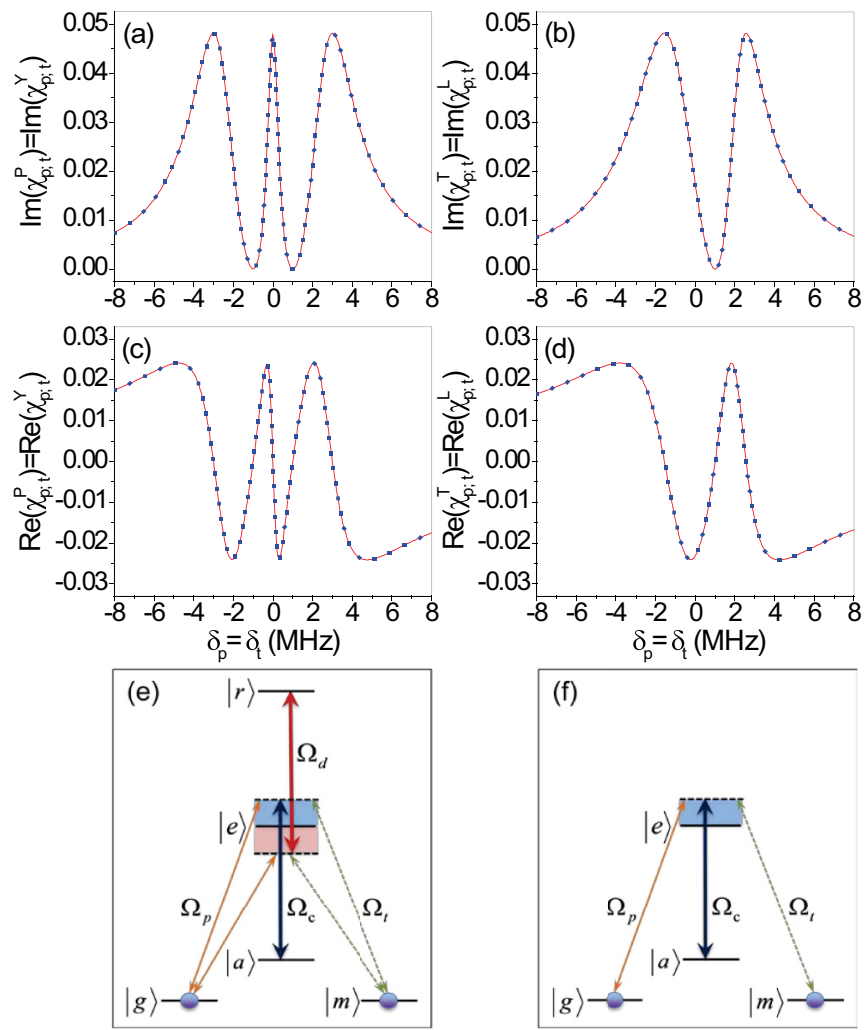

FIG. 2. (Color online) Imaginary $(a, b)$ and real $(c, d)$ parts of probe and trigger susceptibilities vs probe and trigger detunings $\delta_{p}=\delta_{t}$ under a balanced driving (see text) with $\sigma_{g g}=\sigma_{m m}=0.5$ and $\Omega_{p}=\Omega_{t}=3.0 \mathrm{kHz}$. The susceptibilities plotted in (a) and (c) refer to the inverted- $\Psi$ (red-thin line) and inverted-Y (blue-dotted line) configurations whose relevant level transitions are shown schematically in (e). The susceptibilities plotted in (b) and (d) refer to the tripod (red-thin line) and lambda (blue-dotted line) configurations with relevant level transitions shown schematically in (f). The coupling and dressing field parameters are $\Omega_{c}=\Omega_{d}=$ $2.0 \mathrm{MHz}, \delta_{c}=\delta_{d}=1.0 \mathrm{MHz}$ while the atomic parameters are $\gamma_{g e}=$ $\gamma_{m e}=3.0 \mathrm{MHz}, \gamma_{g r}=\gamma_{m r}=3.0 \mathrm{kHz}, \gamma_{g a}=\gamma_{g m}=\gamma_{m a}=1.0 \mathrm{kHz}$, $d_{e g}=d_{e m}=1.5 \times 10^{-29} \mathrm{C} \mathrm{m}$, and $N_{0}=2.4 \times 10^{12} \mathrm{~cm}^{-3}$.

As before they are obtained from Eqs. (3) by setting, respectively, $\Omega_{p}=0$ or $\Omega_{p}=\Omega_{d}=0$.

The analytical results $(A \rightarrow C)$ anticipated above are further discussed here through the direct computation of all relevant atomic susceptibilities. In Fig. 2 we plot both real and imaginary parts of the probe and trigger susceptibilities associated with all six EIT configurations shown in Fig. 1. We first observe that, under a symmetric EIT driving, the probe and trigger fields always exhibit identical susceptibilities whether we deal with an inverted- $\Psi$ (red-thin line) configuration $(A 1)$ or with an inverted-Y (blue-dotted line) configuration $(B 1, C 1)$, that is $\chi_{p}^{P}=\chi_{t}^{P}$ and $\chi_{p}^{Y}=\chi_{t}^{Y}$ as shown in Figs. 2(a) and 2(c). These two panels further show that the probe absorption and dispersion are nearly indistinguishable for an inverted- $\Psi(A 1)$ as well as for an inverted-Y $(B 1, C 1)$ configuration, that is $\chi_{p}^{P} \simeq \chi_{p}^{Y}$. In the same way this holds for the trigger field, with $\chi_{t}^{P} \simeq$ $\chi_{t}^{Y}$. At variance with Refs. $[9,14,36]$, where slightly different probe and trigger detunings are needed to balance 

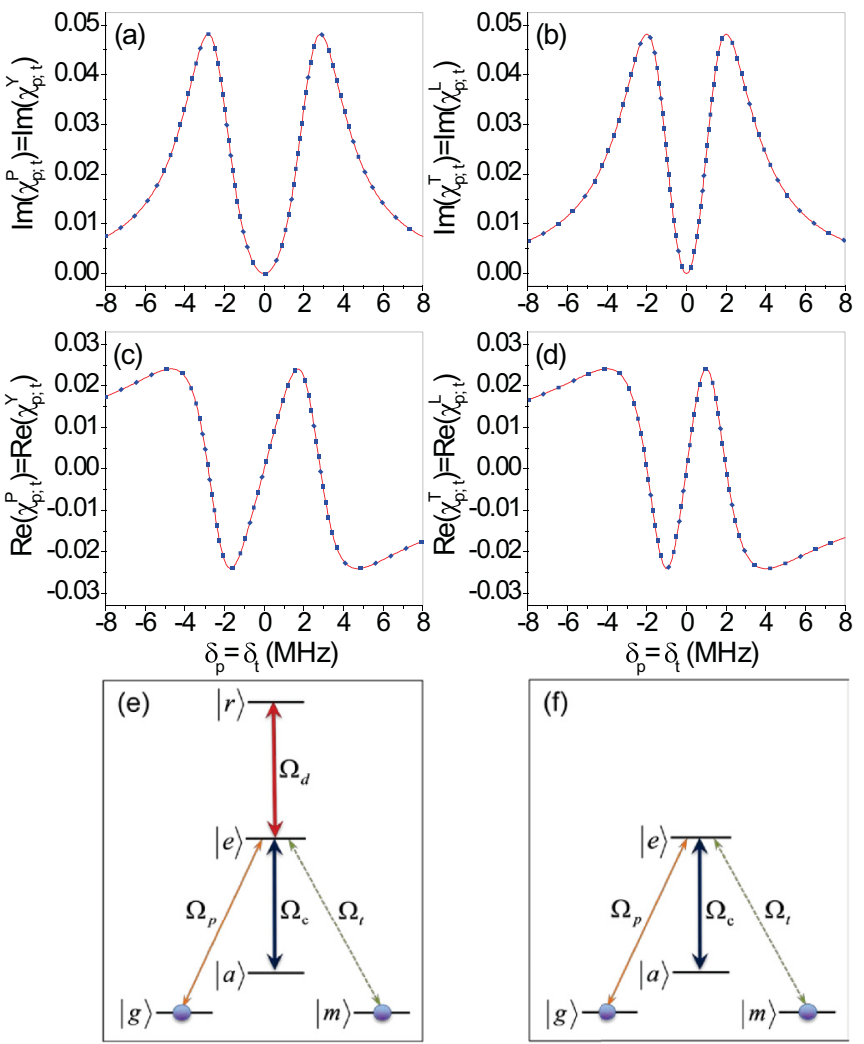

FIG. 3. (Color online) Same as in Fig. 2 except that we take $\delta_{c}=\delta_{d}=0$ here. The pair of probe (trigger) transparency windows now collapse into a single one centered at $\delta_{p}=\delta_{t}=0$ (see text) for the inverted- $\Psi$, inverted- $Y$, tripod, and lambda configurations whose relevant transitions are shown schematically in (e) and (f).

their absorption and dispersion responses, our probe and trigger fields interact in a symmetric configuration with identical detunings and Rabi frequencies. Identical probe and trigger absorption and dispersion are then intrinsic to our model.

Figure 2(a) displays the typical double-EIT spectrum with two probe (trigger) transparency windows placed at $\delta_{p}=\delta_{c}=$ 1.0 MHz and $\delta_{p}=-\delta_{d}=-1.0 \mathrm{MHz}$ and this occurs for both inverted- $\Psi$ and inverted- $Y$ configurations in the nondegenerate near-resonant EIT regime. One transparency window $\left(\delta_{p}=\right.$ $\delta_{c}=1.0 \mathrm{MHz}$ ) opens up as due to the interaction between the longer probe (trigger) leg and the coupling beam, whereas the other window $\left(\delta_{p}=-\delta_{t}=-1.0 \mathrm{MHz}\right)$ arises as due to the interaction of the shorter probe (trigger) leg and the dressing beam as depicted in Fig. 2(e). Such a double-window structure clearly disappears in the presence of dipole Rydberg blockade effects when the three configurations $(A 1-C 1)$ reduce, respectively, to the three configurations $(A 2-C 2)$ [see Fig. 2(f)]. This is observed in Figs. 2(b) and 2(d) where the probe (trigger) susceptibility in the tripod (red-thin line) and lambda (blue-dotted line) configurations clearly exhibits the typical single-EIT spectrum with only one window left at $\delta_{p}=\delta_{c}=1.0 \mathrm{MHz}$. The two equalities mentioned above still hold for the tripod and lambda configurations, namely $\chi_{p}^{T, L}=\chi_{t}^{T, L}$ and $\chi_{p, t}^{T} \simeq \chi_{p, t}^{L}$.
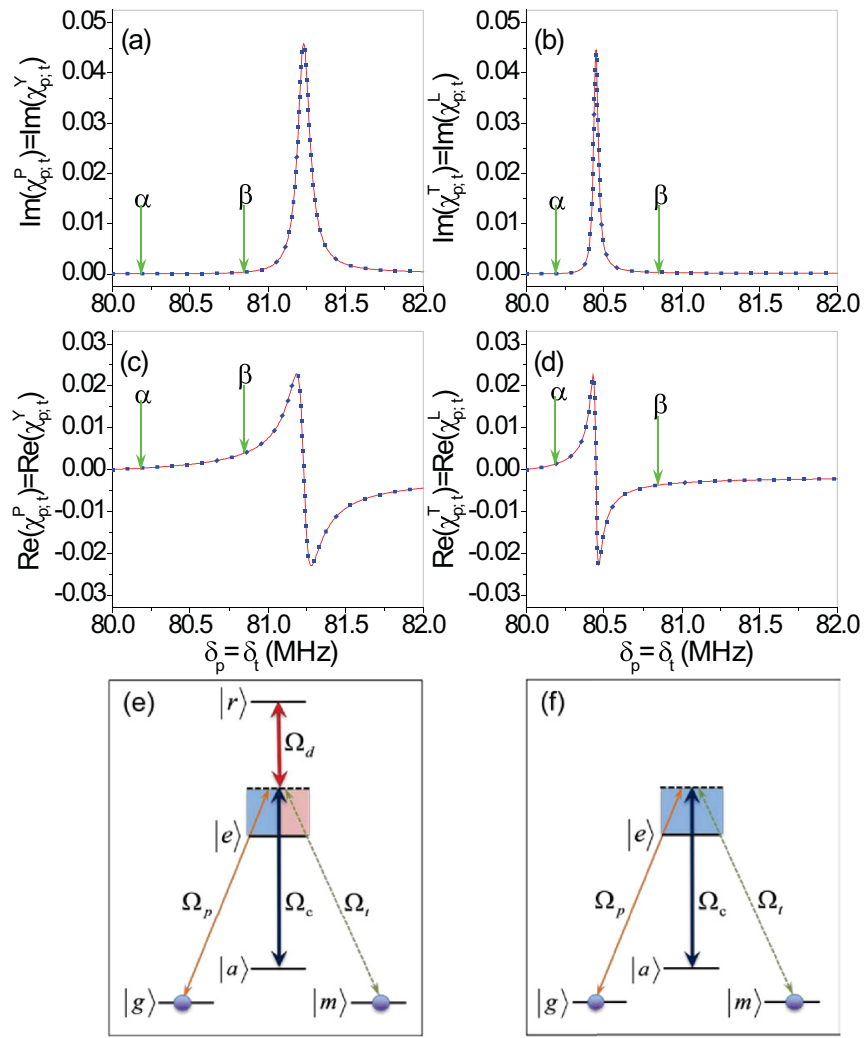

FIG. 4. (Color online) Same as in Fig. 2 except that we take $\delta_{c}=$ $-\delta_{d}=80.0 \mathrm{MHz}, \Omega_{c}=6.0 \mathrm{MHz}$, and $\Omega_{d}=8.0 \mathrm{MHz}$. The pair of probe (trigger) transparency windows now collapse into a single one (see text) centered at $\delta_{p}=\delta_{t}=80.0 \mathrm{MHz}$ for the inverted- $\Psi$, inverted-Y, tripod, and lambda configurations. Points $\alpha$ and $\beta$ denote two different reference detunings used in Sec. V.

Figure 3 is plotted instead for $\delta_{c}=\delta_{d}=0$ (degenerate resonant double-EIT regime) where the two transparency windows collapse into a single one. Notice that in this case the EIT window associated with the inverted- $\Psi$ and inverted- $Y$ configurations is slightly wider than the one observed for the tripod and lambda configurations. Finally, we consider the degenerate far-detuned double-EIT regime with $\delta_{c}=80 \mathrm{MHz}$ and $\delta_{d}=-80 \mathrm{MHz}$. We show in Fig. 4 the absorption line to the right of the center of the transparency window at $\delta_{p}=$ $\delta_{t}=80.0 \mathrm{MHz}$, on a spectral range of detunings of interest for the cross-phase modulation effects discussed in Sec. V. In particular, two specific choices of detuning will be considered below and indicated by points $\alpha$ and $\beta$ in Fig. 4. Such detunings are on the same side of this absorption line [Figs. 4(a) and $4(c)]$ for the inverted- $\Psi$ and inverted- $Y$ configurations. For the corresponding tripod and lambda configurations, instead, points $\alpha$ and $\beta$ are placed on opposite sides with respect to the absorption line [Figs. 4(b) and 4(d)] because the width of the transparency window is reduced as the driving field is decoupled $\left(\Omega_{d} \rightarrow 0\right)$. The $\alpha$ and $\beta$ detunings will be chosen in Sec. V to optimize polarization-selective cross-phase modulation effects, respectively, in a long $(L=2.0 \mathrm{~mm})$ and a short $(L=0.1 \mathrm{~mm})$ sample, while minimizing the losses. 


\section{SA RYDBERG POPULATIONS}

For given polarizations of probe and trigger fields, cold ${ }^{87} \mathrm{Rb}$ atoms under consideration behave in principle as a combination of inverted- $\Psi$ and tripod EIT systems $(A 1 \leftrightarrow A 2)$ or a combination of inverted-Y and lambda EIT systems (B1 $\leftrightarrow B 2$ and $C 1 \leftrightarrow C 2$ ). Such a correlation of level configurations depends critically on dipole blockade of Rydberg excitations, which can be quantified by SA Rydberg populations as discussed below.

Now we transfer to the language of so-called SAs. Each SA is defined by the $n_{\mathrm{SA}} \simeq 4 \pi N_{0} R_{b}^{3} / 3$ atoms inside a blockade sphere of the radius [40]

$$
\begin{aligned}
& R_{b} \simeq\left(\frac{\left|\Delta_{d}\right| C_{6}}{\left|\Omega_{c}\right|^{2}+\left|\Omega_{d}\right|^{2}}\right)^{1 / 6} \text { for }\left|\Delta_{d}\right| \gg \gamma_{g e}=\gamma_{m e}, \\
& R_{b} \simeq\left(\frac{\gamma_{g e} C_{6}}{\left|\Omega_{c}\right|^{2}+\left|\Omega_{d}\right|^{2}}\right)^{1 / 6} \text { for }\left|\Delta_{d}\right| \ll \gamma_{g e}=\gamma_{m e},
\end{aligned}
$$

where the van der Waals (vdW) coefficient $C_{6}$ depends on the principal quantum number $n$ of Rydberg level $|r\rangle$ with $C_{6}=$ $n^{11}\left(c_{0}+c_{1} n+c_{2} n^{2}\right)$ for the $n s-n s$ asymptotes of cold ${ }^{87} \mathrm{Rb}$ atoms [41]. Using $C_{6}=-1.67 \times 10^{13} \mathrm{~s}^{-1} \mu \mathrm{m}^{6}$ for $n=90$, $\Delta_{d}=80 \mathrm{MHz}, \Omega_{c}=6.0 \mathrm{MHz}, \Omega_{d}=8.0 \mathrm{MHz}$, and $N_{0}=$ $2.4 \times 10^{12} \mathrm{~cm}^{-3}$, it is easy to find that the blockade radius is $R_{b} \simeq 15.4 \mu \mathrm{m}$ and the atomic number is $n_{\mathrm{SA}} \simeq 36700$ for a given $\mathrm{SA}$ [42].

\section{A. Polarizations $\left\{\sigma_{p}^{+}, \sigma_{t}^{-}\right\}$}

When the probe and trigger fields exhibit $\sigma_{p}^{+}$and $\sigma_{t}^{-}$polarizations, SAs are described by the ground collective states $|G\rangle=\left|g_{1}, g_{2}, \ldots, g_{n_{\mathrm{SA}} / 2}\right\rangle$ and $|M\rangle=$ $\left|m_{1}, m_{2}, \ldots, m_{n_{\mathrm{SA}} / 2}\right\rangle$ as well as the first-order excited collective states $\left|A^{1}\right\rangle=\left(\left|A^{1 g}\right\rangle+\left|A^{1 m}\right\rangle\right) / \sqrt{2},\left|E^{1}\right\rangle=\left(\left|E^{1 g}\right\rangle+\right.$ $\left.\left|E^{1 m}\right\rangle\right) / \sqrt{2}$, and $\left|R^{1}\right\rangle=\left(\left|R^{1 g}\right\rangle+\left|R^{1 m}\right\rangle\right) / \sqrt{2}$ with the following six components:

$$
\begin{aligned}
\left|R^{1 g}\right\rangle & =\sqrt{\frac{2}{n_{\mathrm{SA}}}} \sum_{j=1}^{n_{\mathrm{SA}} / 2}\left|g_{1}, g_{2}, \ldots, r_{j}, \ldots, g_{n_{\mathrm{SA} / 2}}\right\rangle, \\
\left|E^{1 g}\right\rangle & =\sqrt{\frac{2}{n_{\mathrm{SA}}}} \sum_{j=1}^{n_{\mathrm{SA}} / 2}\left|g_{1}, g_{2}, \ldots, e_{j}, \ldots, g_{n_{\mathrm{SA}} / 2}\right\rangle, \\
\left|A^{1 g}\right\rangle & =\sqrt{\frac{2}{n_{\mathrm{SA}}}} \sum_{j=1}^{n_{\mathrm{SA}} / 2}\left|g_{1}, g_{2}, \ldots, a_{j}, \ldots, g_{n_{\mathrm{SA}} / 2}\right\rangle, \\
\left|R^{1 m}\right\rangle & =\sqrt{\frac{2}{n_{\mathrm{SA}}}} \sum_{j=1}^{n_{\mathrm{SA}} / 2}\left|m_{1}, m_{2}, \ldots, r_{j}, \ldots, m_{n_{\mathrm{SA}} / 2}\right\rangle, \\
\left|E^{1 m}\right\rangle & =\sqrt{\frac{2}{n_{\mathrm{SA}}}} \sum_{j=1}^{n_{\mathrm{SA}} / 2}\left|m_{1}, m_{2}, \ldots, e_{j}, \ldots, m_{n_{\mathrm{SA} / 2}}\right\rangle, \\
\left|A^{1 m}\right\rangle & =\sqrt{\frac{2}{n_{\mathrm{SA}}}} \sum_{j=1}^{n_{\mathrm{SA}} / 2}\left|m_{1}, m_{2}, \ldots, a_{j}, \ldots, m_{n_{\mathrm{SA} / 2}}\right\rangle,
\end{aligned}
$$

while other higher-order excited collective states (with more than one atoms at level $|r\rangle$ or level $|a\rangle$ ) can be neglected near the EIT window center [35].
The SA operators $\Sigma_{i j}=|I\rangle\langle J|$ obey similar dynamic equations as those for atomic operators $\sigma_{i j}=|i\rangle\langle j|$ except that the probe and trigger Rabi frequencies should be respectively replaced by [43]

$$
\Omega_{p} \rightarrow \Omega_{p}^{s}=\sqrt{\frac{n_{\mathrm{SA}}}{2}} \Omega_{p}, \quad \Omega_{t} \rightarrow \Omega_{t}^{s}=\sqrt{\frac{n_{\mathrm{SA}}}{2}} \Omega_{t} .
$$

That is, the collective transitions $|G\rangle \leftrightarrow\left|E^{1 g}\right\rangle$ and $|M\rangle \leftrightarrow$ $\left|E^{1 m}\right\rangle$ are enhanced to have, respectively, Rabi frequencies $\Omega_{p}^{s}$ and $\Omega_{t}^{s}$, which may be comparable to $\Omega_{c}$ and $\Omega_{d}$ even if $\Omega_{p, t} \ll$ $\Omega_{c, d}$. Note, however, that dipole-dipole interactions may result in the modification of two-particle quantum correlations. As a matter of fact [30], $\Omega_{p, t}^{2}$ should be further replaced by $\Omega_{p, t}^{2} g_{p, t}$ to include the two-photon correlations

$$
g_{p, t}=\frac{\left\langle\hat{\Omega}_{p, t}^{\dagger} \hat{\Omega}_{p, t}^{\dagger} \hat{\Omega}_{p, t} \hat{\Omega}_{p, t}\right\rangle}{\left\langle\hat{\Omega}_{p, t}^{\dagger} \hat{\Omega}_{p, t}\right\rangle^{2}}
$$

quantifying the probability of having at least two photons in a blockade volume, but we have $g_{p, t} \rightarrow 1.0$ near the center of a degenerate EIT window at $\delta_{p, t}=-\delta_{d}=\delta_{c}$ with $\left|\delta_{c, d}\right| \gg$ $\gamma_{g e}$ when the input probe and trigger fields are in coherent states [35]. Thus we obtain from Eqs. (3)

$$
\begin{aligned}
\partial_{t} \Sigma_{g e}^{P}= & -\gamma_{g e}^{\prime} \Sigma_{g e}^{P}+i \Omega_{d}^{*} \Sigma_{g r}^{P} \\
& +i \Omega_{c} \Sigma_{g a}^{P}+i \Omega_{t}^{s} \Sigma_{g m}^{P}+i \Omega_{p}^{s} \Sigma_{g g}^{P}, \\
\partial_{t} \Sigma_{g a}^{P}= & -\gamma_{g a}^{\prime} \Sigma_{g a}^{P}+i \Omega_{c}^{*} \Sigma_{g e}^{P}, \\
\partial_{t} \Sigma_{g r}^{P}= & -\gamma_{g r}^{\prime} \Sigma_{g r}^{P}+i \Omega_{d} \Sigma_{g e}^{P}, \\
\partial_{t} \Sigma_{m e}^{P}= & -\gamma_{m e}^{\prime} \Sigma_{m e}^{P}+i \Omega_{d}^{*} \Sigma_{m r}^{P}+i \Omega_{c} \Sigma_{m a}^{P} \\
& +i \Omega_{p}^{s} \Sigma_{m g}^{P}+i \Omega_{t}^{s} \Sigma_{m m}^{P}, \\
\partial_{t} \Sigma_{m a}^{P}= & -\gamma_{m a}^{\prime} \Sigma_{m a}^{P}+i \Omega_{c}^{*} \Sigma_{m e}^{P}, \\
\partial_{t} \Sigma_{m r}^{P}= & -\gamma_{m r}^{\prime} \Sigma_{m r}^{P}+i \Omega_{d} \Sigma_{m e}^{P}, \\
\partial_{t} \Sigma_{g m}^{P}= & -\gamma_{g m}^{\prime} \Sigma_{g m}^{P}+i \Omega_{t}^{s \dagger} \Sigma_{g e}^{P}-i \Omega_{p}^{s} \Sigma_{e m}^{P},
\end{aligned}
$$

for the inverted- $\Psi$ double-EIT system. To solve Eqs. (14) in the steady state, we should first express $\Sigma_{g g}^{P}$ and $\Sigma_{m m}^{P}$ in terms of the variables $\Sigma_{i j}^{P}$ and three considerations are now in order. First, we recall that

$$
\begin{gathered}
\Sigma_{a a}^{P}=\Sigma_{a a}^{g P}+\Sigma_{a a}^{m P}=\frac{\Sigma_{a g}^{P} \Sigma_{g a}^{P}}{\Sigma_{g g}^{P}}+\frac{\Sigma_{a m}^{P} \Sigma_{m a}^{P}}{\Sigma_{m m}^{P}}, \\
\Sigma_{e e}^{P}=\Sigma_{e e}^{g P}+\Sigma_{e e}^{m P}=\frac{\Sigma_{e g}^{P} \Sigma_{g e}^{P}}{\Sigma_{g g}^{P}}+\frac{\Sigma_{e m}^{P} \Sigma_{m e}^{P}}{\Sigma_{m m}^{P}}, \\
\Sigma_{r r}^{P}=\Sigma_{r r}^{g P}+\Sigma_{r r}^{m P}=\frac{\Sigma_{r g}^{P} \Sigma_{g r}^{P}}{\Sigma_{g g}^{P}}+\frac{\Sigma_{r m}^{P} \Sigma_{m r}^{P}}{\Sigma_{m m}^{P}},
\end{gathered}
$$

and second, we notice that $\Sigma_{a a}^{P}$ and $\Sigma_{r r}^{P}$ may be comparable to $\Sigma_{g g}^{P}$ and $\Sigma_{m m}^{P}$, as a result of enhanced collective transitions, whereas $\Sigma_{e e}^{P}$ is negligible owing to quantum destructive interference in the EIT regime. In fact, $\Sigma_{a a}^{g P} / \Sigma_{r r}^{g P} \approx \Omega_{c}^{2} / \Omega_{d}^{2}$ and $\Sigma_{e e}^{g P} / \Sigma_{r r}^{g P} \approx \gamma_{g r}^{2} / \Omega_{d}^{2}$ in the two-photon near-resonant case of $\left|\Delta_{a, r}\right| \ll \Omega_{c, d}$ as well as $\Sigma_{a a}^{m P} / \Sigma_{r r}^{m P} \approx \Omega_{c}^{2} / \Omega_{d}^{2}$ and $\Sigma_{e e}^{m P} / \Sigma_{r r}^{m P} \approx \gamma_{m r}^{2} / \Omega_{d}^{2}$ in the two-photon near-resonant case of $\left|\Delta_{a, r}^{\prime}\right| \ll \Omega_{c, d}$, as follows from Eqs. (14) in the steady 
state. Third, notice that $\Sigma_{r r}^{g P}+\Sigma_{a a}^{g P}+\Sigma_{g g}^{P}=0.5$ and $\Sigma_{r r}^{m P}+$ $\Sigma_{a a}^{m P}+\Sigma_{m m}^{P}=0.5$ in a good approximation for the boundary conditions $\Omega_{p}(z=0)=\Omega_{t}(z=0)$ and initial conditions
$\Sigma_{g g}(t=0)=\Sigma_{m m}(t=0)$, as follows from the second consideration and the conservation of the SA population. From all the above we obtain

$$
\Sigma_{g g}^{P}=\frac{1}{4}+\frac{1}{4} \sqrt{1-16\left(\Sigma_{r g}^{P} \Sigma_{g r}^{P}+\Sigma_{a g}^{P} \Sigma_{g a}^{P}\right)}, \quad \Sigma_{m m}^{P}=\frac{1}{4}+\frac{1}{4} \sqrt{1-16\left(\Sigma_{r m}^{P} \Sigma_{m r}^{P}+\Sigma_{a m}^{P} \Sigma_{m a}^{P}\right)},
$$

which are then applied to solve Eqs. (14) upon setting $\partial_{t} \Sigma_{i j}^{P}=0$ to arrive at the SA Rydberg populations

$$
\begin{aligned}
\Sigma_{r r}^{g P} & =\frac{0.5\left|\gamma_{g a}^{\prime}\right|^{2}\left|\Omega_{p}^{s}\right|^{2}\left|\Omega_{d}\right|^{2}}{\left.\left|A_{g}+\gamma_{m r}^{\prime *}\left(\gamma_{m a}^{\prime *} / \gamma_{g m}^{\prime}\right)\right| \Omega_{p}^{s}\right|^{2}\left(A_{g} / A_{m}^{*}\right)+\left.\gamma_{g r}^{\prime}\left(\gamma_{g a}^{\prime} / \gamma_{g m}^{\prime}\right)\left|\Omega_{t}^{s}\right|^{2}\right|^{2}+\left(\gamma_{g a}^{\prime 2}\left|\Omega_{d}\right|^{2}+\gamma_{g r}^{\prime 2}\left|\Omega_{c}\right|^{2}\right)\left|\Omega_{p}^{s}\right|^{2}}, \\
\Sigma_{r r}^{m P} & =\frac{0.5\left|\gamma_{m a}^{\prime}\right|^{2}\left|\Omega_{t}^{s}\right|^{2}\left|\Omega_{d}\right|^{2}}{\left.\left|A_{m}+\gamma_{g r}^{\prime *}\left(\gamma_{g a}^{\prime *} / \gamma_{m g}^{\prime}\right)\right| \Omega_{t}^{s}\right|^{2}\left(A_{m} / A_{g}^{*}\right)+\left.\gamma_{m r}^{\prime}\left(\gamma_{m a}^{\prime} / \gamma_{m g}^{\prime}\right)\left|\Omega_{p}^{s}\right|^{2}\right|^{2}+\left(\gamma_{m a}^{\prime 2}\left|\Omega_{d}\right|^{2}+\gamma_{m r}^{\prime 2}\left|\Omega_{c}\right|^{2}\right)\left|\Omega_{t}^{s}\right|^{2}},
\end{aligned}
$$

for the inverted- $\Psi$ double-EIT system.

\section{B. Polarizations $\left\{\sigma_{p}^{+}, \sigma_{t}^{+}\right\}\left(\left\{\sigma_{p}^{-}, \sigma_{t}^{-}\right\}\right)$}

When the probe and trigger fields exhibit $\sigma_{p}^{+}$and $\sigma_{t}^{+}$(or $\sigma_{p}^{-}$and $\sigma_{t}^{-}$) polarizations, SAs are described by the collective states $|G\rangle,\left|A^{1 g}\right\rangle,\left|E^{1 g}\right\rangle$, and $\left|R^{1 g}\right\rangle$ (or $|M\rangle,\left|A^{1 m}\right\rangle,\left|E^{1 m}\right\rangle$, and $\left.\left|R^{1 m}\right\rangle\right)$ in the inverted-Y configuration. In this case, relevant SA Liouville equations can be derived by setting $\Omega_{t}^{s}=0$ or $\Omega_{p}^{s}=$ 0 in Eqs. (14). Then with similar considerations mentioned above, we obtain the SA Rydberg populations:

$$
\begin{aligned}
\Sigma_{r r}^{g Y} & =\frac{0.5\left|\gamma_{g a}^{\prime}\right|^{2}\left|\Omega_{p}^{s}\right|^{2}\left|\Omega_{d}\right|^{2}}{\left|A_{g}\right|^{2}+\left(\left|\gamma_{g a}^{\prime}\right|^{2}\left|\Omega_{d}\right|^{2}+\left|\gamma_{g r}^{\prime}\right|^{2}\left|\Omega_{c}\right|^{2}\right)\left|\Omega_{p}^{s}\right|^{2}}, \\
\Sigma_{r r}^{m Y} & =\frac{0.5\left|\gamma_{m a}^{\prime}\right|^{2}\left|\Omega_{t}^{s}\right|^{2}\left|\Omega_{d}\right|^{2}}{\left|A_{m}\right|^{2}+\left(\left|\gamma_{m a}^{\prime}\right|^{2}\left|\Omega_{d}\right|^{2}+\left|\gamma_{m r}^{\prime}\right|^{2}\left|\Omega_{c}\right|^{2}\right)\left|\Omega_{t}^{s}\right|^{2}},
\end{aligned}
$$

for the inverted- $Y$ double-EIT systems. In a good approximation, they are restricted by $\Sigma_{r r}^{g Y}+\Sigma_{a a}^{g Y}+\Sigma_{g g}^{Y}=0.5$ and $\Sigma_{r r}^{m Y}+\Sigma_{a a}^{m Y}+\Sigma_{m m}^{Y}=0.5$, respectively.

In Figs. 5 and 6 we plot SA Rydberg populations in the inverted- $\Psi$ and inverted- $Y$ configurations as a function of probe and trigger detunings $\delta_{p}=\delta_{t}$, for realistic values of $\Omega_{p}=\Omega_{t}$ and of $\gamma_{g r}=\gamma_{m r}$. Figure 5 is attained in the degenerate resonant EIT regime with $\delta_{c}=-\delta_{d}=0$, while Fig. 6 is attained in the degenerate far-detuned EIT regime with $\delta_{c}=-\delta_{d}=80 \mathrm{MHz}$. We observe a remarkable difference of SA Rydberg populations $\Sigma_{r r}^{g P}-\Sigma_{r r}^{g Y}\left(\Sigma_{r r}^{m P}-\Sigma_{r r}^{m Y}\right)$ even if $\Omega_{p, t} \ll \Omega_{c, d}$ since it is possible to have $\Omega_{p, t}^{s} \sim \Omega_{c, d}$ in a dense enough sample. We also observe $\Sigma_{r r}^{g Y}=\Sigma_{r r}^{m Y} \gg$ $\Sigma_{r r}^{g P}=\Sigma_{r r}^{m P}$, indicating that SA Rydberg excitations are large when only the probe or trigger field is coupled to the $|e\rangle$ level, while they are suppressed when both probe and trigger fields are coupled to the $|e\rangle$ level. This is a salient result as it prompts a remarkable difference between the SA Rydberg populations, respectively, in the inverted- $\Psi$ and inverted-Y configurations, which might be exploited to attain large conditional phase shifts [44,45]. Such an essential difference basically arises from the facts that (i) the collective SA transition amplitudes $|G\rangle \leftrightarrow\left|E^{1 g}\right\rangle$ and
$|M\rangle \leftrightarrow\left|E^{1 m}\right\rangle$ are both enhanced by the large factor $\sqrt{n_{s a} / 2}$ and (ii) in the symmetric inverted- $\Psi$ configuration $(A 1)$ the two transition pathways interfere destructively yielding a greatly suppressed Rydberg population compared to those observed in the asymmetric inverted- $Y$ configurations ( $B 1$ and $C 1$ ), encompassing instead only a single enhanced transition amplitude.

It should be finally noticed that in the limit of weak probe and trigger fields, even when dipole blockade is effective, the actual fraction of atoms in levels different from $|g\rangle$ and $|m\rangle$ remains very small: even for SA Rydberg populations of order 1.0 as in Figs. 5 and $6, \sigma_{e e}<\sigma_{a a} \sim \sigma_{r r}<10^{-4}$ as $\sigma_{i i} \sim \Sigma_{I I} / n_{\mathrm{SA}}$ and $n_{\mathrm{SA}} \sim 36000$. Then, heating of a sample due to absorption of coupling and dressing fields is strongly suppressed as they both interact with nearly empty levels.
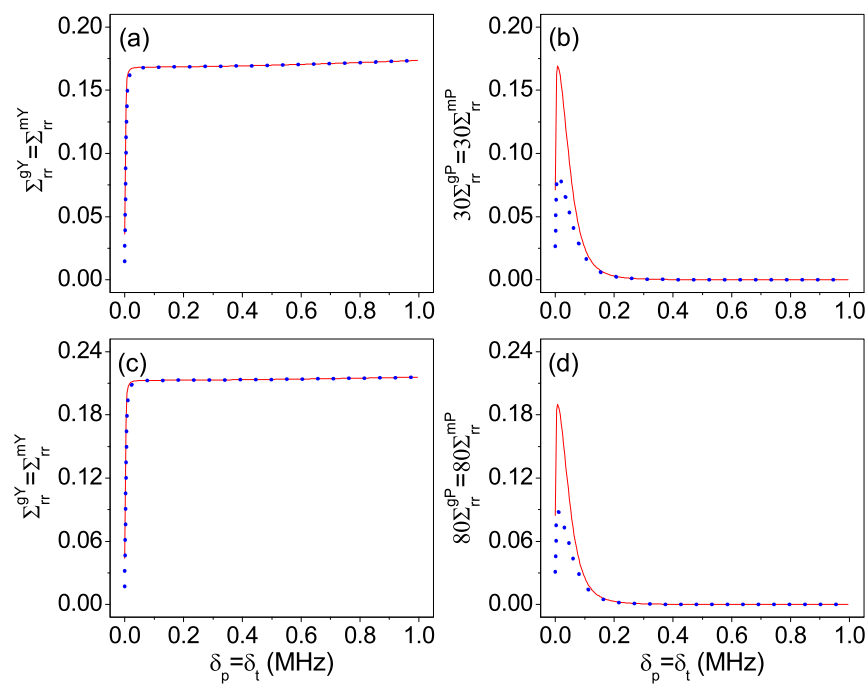

FIG. 5. (Color online) SA Rydberg populations for the inverted$\mathrm{Y}(\mathrm{a}, \mathrm{c})$ and inverted $-\Psi$ (b, d) configurations in the degenerate resonant double-EIT regime with $\delta_{c}=-\delta_{d}=0$. Red-thin and blue-dotted curves correspond, respectively, to $\gamma_{g r}=\gamma_{m r}=3.0 \mathrm{kHz}$ and $\gamma_{g r}=$ $\gamma_{m r}=5.0 \mathrm{kHz}$. Other parameters are the same as in Fig. 3 except $\Omega_{p}=\Omega_{t}=30 \mathrm{kHz}$ in (a) and (b); $\Omega_{p}=\Omega_{t}=50 \mathrm{kHz}$ in (c) and (d). 

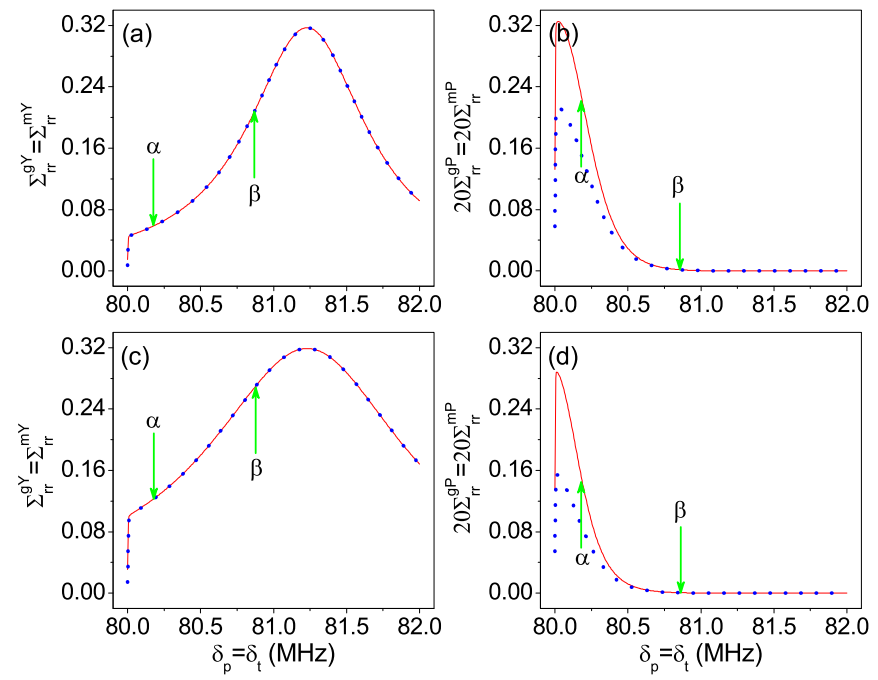

FIG. 6. (Color online) SA Rydberg populations for the inverted$Y(a, c)$ and inverted- $\Psi$ (b, d) configurations in the degenerate fardetuned double-EIT regime with $\delta_{c}=-\delta_{d}=80 \mathrm{MHz}$. Red-thin and blue-dotted curves refer, respectively, to $\gamma_{g r}=\gamma_{m r}=3.0 \mathrm{kHz}$ and $\gamma_{g r}=\gamma_{m r}=5.0 \mathrm{kHz}$. Other parameters are the same as in Fig. 4 except $\Omega_{p}=\Omega_{t}=30 \mathrm{kHz}$ in (a) and (b); $\Omega_{p}=\Omega_{t}=50 \mathrm{kHz}$ in (c) and (d).

\section{RYDBERG COOPERATIVE NONLINEARITIES}

With the individual susceptibilities of Sec. III and the SA populations of Sec. IV, we now examine the dispersion and absorption properties exhibited by a pair of monochromatic probe and trigger fields traveling through an atomic sample in the polarization configurations of Fig. 1. For each of the correlated EIT regimes $A 1 \leftrightarrow A 2, B 1 \leftrightarrow B 2$, and $C 1 \leftrightarrow C 2$, the probe and trigger susceptibilities can be written as

$$
\begin{aligned}
& \chi_{p}^{a}=2 \Sigma_{r r}^{g P} \chi_{p}^{T}+\left(1-2 \Sigma_{r r}^{g P}\right) \chi_{p}^{P} \quad \text { for } \quad\left\{\sigma_{p}^{+}, \sigma_{t}^{-}\right\}, \\
& \chi_{t}^{a}=2 \Sigma_{r r}^{m P} \chi_{t}^{T}+\left(1-2 \Sigma_{r r}^{m P}\right) \chi_{t}^{P} \quad \text { for } \quad\left\{\sigma_{p}^{+}, \sigma_{t}^{-}\right\}, \\
& \chi_{p}^{b}=2 \Sigma_{r r}^{g Y} \chi_{p}^{L}+\left(1-2 \Sigma_{r r}^{g Y}\right) \chi_{p}^{Y} \text { for }\left\{\sigma_{p}^{+}, \sigma_{t}^{+}\right\}, \\
& \chi_{t}^{c}=2 \Sigma_{r r}^{m Y} \chi_{t}^{L}+\left(1-2 \Sigma_{r r}^{m Y}\right) \chi_{t}^{Y} \text { for }\left\{\sigma_{p}^{-}, \sigma_{t}^{-}\right\},
\end{aligned}
$$

clearly showing cooperative dispersion and absorption effects depending on whether or not the sample is optically driven into the Rydberg state (dipole blockade). The concept of a blockade sphere for Rydberg excitation, adopted here [46,47] to get an intuitive physical picture on real effects of the dipoledipole interaction on the probe and trigger coherences (2), enables one to include Rydberg blockade in the average atomic susceptibilities (18)-(21) which will then be used to describe the optical response of the entire sample. A monochromatic probe impinging upon a sample of length $L$ with a vacuum wave-vector $k_{p}$ experiences, e.g., the phase shift

$$
\phi_{p}^{a, b}=\operatorname{Re}\left(\chi_{p}^{a, b}\right) k_{p} L / 2,
$$

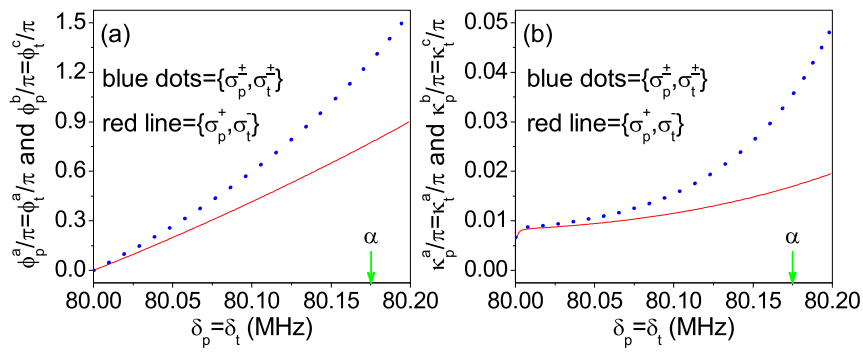

FIG. 7. (Color online) Cooperative phase shifts (a) and extinction coefficients (b) acquired over a $L=2.0 \mathrm{~mm}$ long sample around the point $\alpha$ (Fig. 4) under the inverted- $\Psi$ ( $A 1$ ) (red-thin line) and inverted-Y $(B 1, C 1)$ (blue-dotted line) configurations. Here $\gamma_{g r}=$ $\gamma_{m r}=3.0 \mathrm{kHz}$ and $\Omega_{p}=\Omega_{t}=50 \mathrm{kHz}$ while other parameters are the same as in Fig. 6.

whereas the extinction coefficient is

$$
\kappa_{p}^{a, b}=\operatorname{Im}\left(\chi_{p}^{a, b}\right) k_{p} L / 2 .
$$

Similar expressions for $\phi_{t}^{a, c}$ and $\kappa_{t}^{a, c}$ hold for the trigger upon replacing $p \rightarrow t$ in the above equations. Because the susceptibilities $\chi_{p ; t}^{P}\left(\chi_{p ; t}^{T}\right)$ and $\chi_{p ; t}^{Y}\left(\chi_{p ; t}^{L}\right)$ are indistinguishable (see Fig. 4) and the populations $\Sigma_{r r}^{g P}\left(\Sigma_{r r}^{m P}\right)$ and $\Sigma_{r r}^{g Y}\left(\Sigma_{r r}^{m Y}\right)$ are in general very different (see Fig. 6), we expect that $\phi_{p}^{a}\left(\kappa_{p}^{a}\right)$ be different from $\phi_{p}^{b}\left(\kappa_{p}^{b}\right)$ and likewise $\phi_{t}^{a}\left(\kappa_{t}^{a}\right)$ be different from $\phi_{t}^{c}\left(\kappa_{t}^{c}\right)$. Such departures are then a direct manifestation of the cooperative nonlinearities arising from the dipole Rydberg blockade. When Rydberg transitions are involved, in fact, the steady-state optical response, hence the phase shifts and absorption, will also depend on the probability to find an atom excited into the Rydberg state.

We plot in Figs. 7 and 8 the cooperative phase shifts $\phi_{p}^{a, b}=$ $\phi_{t}^{a, c}$ and extinction coefficients $\kappa_{p}^{a, b}=\kappa_{t}^{a, c}$ as a function of probe and trigger detunings $\delta_{p}=\delta_{t}$. This is done, respectively, for a long sample $(L=2.0 \mathrm{~mm})$ containing about $65 \mathrm{SAs}$ around point $\alpha$ and for a short sample $(L=0.1 \mathrm{~mm})$ containing only about 3 SAs around point $\beta$ in the degenerate far-detuned regime with $\delta_{c}=-\delta_{d}=80 \mathrm{MHz}$ (see Figs. 4 and 6). We can reach $\phi_{p}^{a}=\phi_{t}^{a}=0.77 \pi$ and $\phi_{p}^{b}=\phi_{t}^{c}=1.27 \pi$, with $\kappa_{p}^{a}=$ $\kappa_{t}^{a} \approx 0.02 \pi$ and $\kappa_{p}^{b}=\kappa_{t}^{c} \approx 0.04 \pi$ at $\delta_{p}=\delta_{t} \approx 80.17 \mathrm{MHz}$ (point $\alpha$ ) in Fig. 7. We further observe $\phi_{p}^{a}=\phi_{t}^{a}=0.48 \pi$ and $\phi_{p}^{b}=\phi_{t}^{c}=-0.01 \pi$, with $\kappa_{p}^{a}=\kappa_{t}^{a} \approx 0.04 \pi$ and $\kappa_{p}^{b}=\kappa_{t}^{c} \approx$ $0.04 \pi$ at $\delta_{p}=\delta_{t} \approx 80.850 \mathrm{MHz}$ (point $\beta$ ) in Fig. 8 .
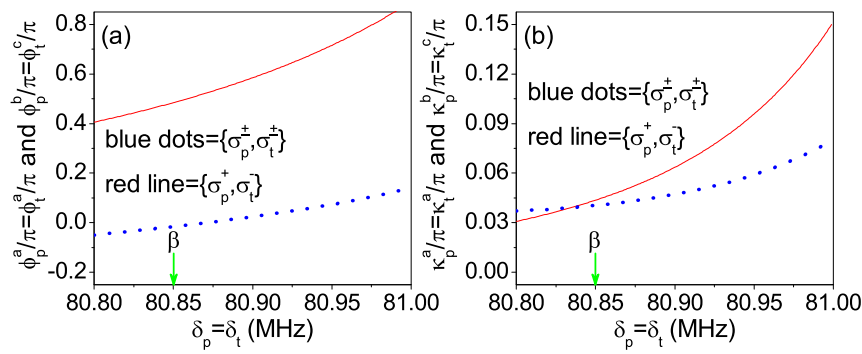

FIG. 8. (Color online) Same as in Fig. 7 for phase shifts (a) and extinction coefficients (b) acquired over a $L=0.1 \mathrm{~mm}$ short sample around the point $\beta$ (Fig. 4). 

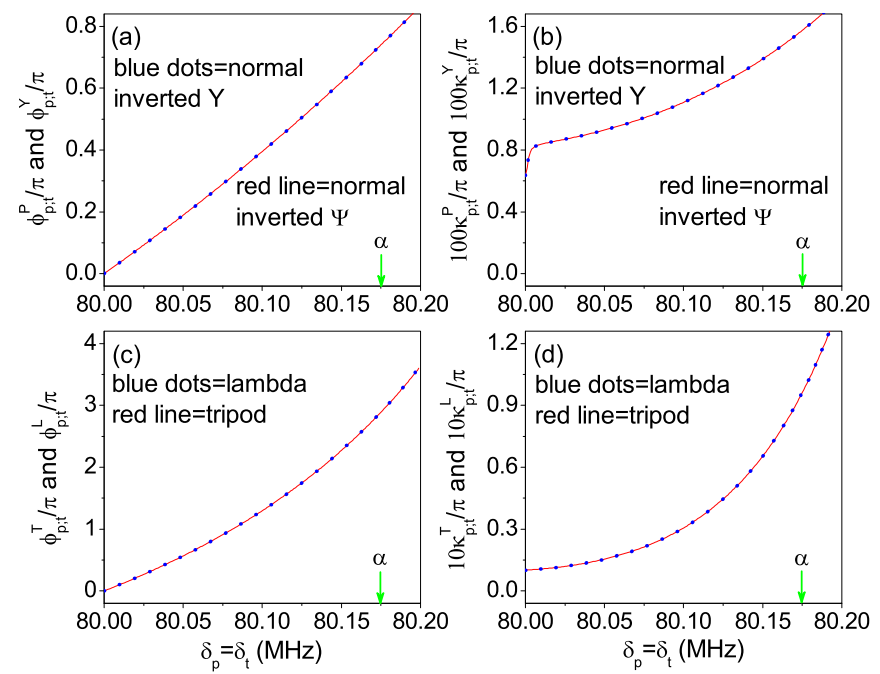

FIG. 9. (Color online) Same as in Fig. 7 where phase shifts (a) and extinction coefficients (b) are acquired under the inverted- $\Psi$ $(A 1)$ (red-thin line) and inverted-Y $(B 1, C 1)$ (blue-dotted line) driving configurations, with $|r\rangle$ being a normal excited state and where the phase shifts (c) and extinction coefficients (d) are acquired under the tripod $(A 2)$ (red-thin line) and lambda $(B 2, C 2)$ (blue-dotted line) driving configurations, in the absence of Rydberg excitation to the state $|r\rangle$.

The cooperative nonlinearity arising from conditional Rydberg blockade may finally be compared with those arising from standard cross-Kerr EIT processes [2-4,36] and to this extent we examine two specific cases. We take (i) a sample driven into the inverted- $\Psi$ and inverted- $Y$ double-EIT configurations, yet with $|r\rangle$ being a normal excited level and (ii) a sample driven into the tripod and lambda single-EIT configurations. In case (i), the probe and trigger phase shifts reduce to $\phi_{p, t}^{P}=\operatorname{Re}\left(\chi_{p, t}^{P}\right) k_{p, t} L / 2$ and $\phi_{p, t}^{Y}=\operatorname{Re}\left(\chi_{p, t}^{Y}\right) k_{p, t} L / 2$, while the extinction constants reduce to $\kappa_{p, t}^{P}=\operatorname{Im}\left(\chi_{p, t}^{P}\right) k_{p, t} L / 2$ and $\kappa_{p, t}^{Y}=\operatorname{Im}\left(\chi_{p, t}^{Y}\right) k_{p, t} L / 2$. In case (ii), on the other hand, we have for the phase shifts $\phi_{p, t}^{T}=\operatorname{Re}\left(\chi_{p, t}^{T}\right) k_{p, t} L / 2$ and $\phi_{p, t}^{L}=\operatorname{Re}\left(\chi_{p, t}^{L}\right) k_{p, t} L / 2$ and for the extinction constants $\kappa_{p, t}^{T}=\operatorname{Im}\left(\chi_{p, t}^{T}\right) k_{p, t} L / 2$ and $\kappa_{p, t}^{L}=\operatorname{Im}\left(\chi_{p, t}^{L}\right) k_{p, t} L / 2$. This is shown in Fig. 9 where we use the same parameters as in Fig. 7 and plot in the left (right) panels the individual phase shifts $\phi_{p}^{P, Y}=\phi_{t}^{P, Y}\left(\phi_{p}^{T, L}=\phi_{t}^{T, L}\right)$ and individual extinction constants $\kappa_{p}^{P, Y}=\kappa_{t}^{P, Y}\left(\kappa_{p}^{T, L}=\kappa_{t}^{T, L}\right)$ around point $\alpha$ [48]. Similar levels of absorptive losses and of (absolute) phase shifts are observed in Fig. 9. Yet unlike in Fig. 7, there are no appreciable differences between the two choices $\left\{\sigma_{p}^{+}, \sigma_{t}^{-}\right\}$ (both probe and trigger coupled) and $\left\{\sigma_{p}^{ \pm}, \sigma_{t}^{ \pm}\right\}$(either probe or trigger coupled) for probe and trigger polarizations. This indicates that the conditional Rydberg blockade can be exploited to attain cross-phase modulation effects that are in general hard to achieve with the usual Kerr-EIT nonlinearities.

\section{CONCLUSIONS}

A pair of weak probe and trigger fields interacting with a pair of strong coupling and dressing fields through a dense sample of cold rubidium atoms that may be driven to a high Rydberg state can experience diverse EIT regimes depending on (a) the probe and trigger polarizations and (b) the presence of Rydberg blockade effects. This hinges on the probe and trigger circular polarization selection rules and on the strength of the linearly polarized coupling and dressing fields, a most relevant instance of which is illustrated in the five-level inverted- $\Psi$ EIT configuration $(A 1)$ of Fig. 1 . The optical response in this configuration is central to this work and enables us, in turn, to readily compute the optical responses of other (reduced) EIT configurations, i.e., inverted-Y, tripod, and lambda configurations (Sec. III). In each of these EIT configurations, whether Rydberg blockade is present or not, the probe and trigger propagation can be engineered so as to occur without significant absorption and dispersion. Depending on the choice of circular polarizations, on the other hand, probe and trigger cooperative cross nonlinear interactions originating from Rydberg blockade become much stronger than those observed in typical Kerr-EIT media yet at the same levels of linear absorption and dispersion [2-4,36].

Our inverted- $\Psi$ configuration, characterized by large polarization conditional nonlinear interactions between two weak optical fields with little absorption and dispersion, allows for efficient cross-phase modulation effects. The required conditions are (i) a degenerate EIT regime $\left(\delta_{c}=-\delta_{d}\right)$ with strong enough coupling and dressing fields $\left(\Omega_{c, d} \sim \gamma_{g e} \sim\right.$ $\left.\gamma_{m e}\right)$, (ii) far off-resonant probe and trigger EIT windows $\left(\delta_{p}=\delta_{t}=\delta_{c}=-\delta_{d}=80 \mathrm{MHz}\right.$, in our case) to guarantee that the two-photon correlation functions $g_{p} \approx g_{t} \approx 1$, and finally (iii) a symmetric driving with equally populated ground levels $|g\rangle$ and $|m\rangle$. The last requirement is not only beneficial to achieve a matching of probe and trigger fields but also to ensure a suppression of SA Rydberg populations for the polarization choice of Fig. 1(a), in comparison with those of Figs. 1(b) and 1(c). One key advantage of our scheme is that Rydberg blockade results in the conditional reduction from a double EIT configuration to a simple EIT one, rather than to an absorbing one. This is the critical point for suppressing linear absorption and dispersion for any choices of probe and trigger (circular) polarizations.

The scheme we propose may be relevant to a variety of all-optical polarization-sensitive cross-phase nonlinearities with conditional phase shifts on the order of $\pi$. Dipole blockade depends on the probe and trigger polarizations with the inverted $-\Psi$ and inverted- $Y$ configurations exhibiting, in fact, quite different Rydberg populations. In particular, the scheme we propose could provide the basic physical mechanism for a universal vector gate [14] where the nearly lossless and distortionless propagation of probe and trigger pulses would be naturally matched thanks to the symmetry of our level configuration. Large values of cross phase shifts could be attained not only in samples containing only a few rubidium SAs, but also employing very weak light pulses. We anticipate that cross phase shifts of the order of $\pi$ might be achieved with light pulses containing only a few hundreds of photons, the reason being that the variation of SA Rydberg populations is mainly determined by $n_{s a} \Omega_{p, t}^{2}$ so that very small Rabi frequencies $\Omega_{p, t}$ could be compensated by a large number $n_{s a}$. 


\section{ACKNOWLEDGMENTS}

This work is supported by the National Natural Science Foundation (No. 11174110 and No. 61378094), the
National Basic Research Program (No. 2011CB921603), and the 111 Project (No. B13013) of China. Jin-Hui Wu acknowledges the hospitality of Scuola Normale Superiore at Pisa.
[1] P. Kok, W. J. Munro, K. Nemoto, T. C. Ralph, J. P. Dowling, and G. J. Milburn, Rev. Mod. Phys. 79, 135 (2007).

[2] H. Schmidt and A. Imamoglu, Opt. Lett. 21, 1936 (1996).

[3] S.-J. Li, X.-D. Yang, X.-M. Cao, C.-H. Zhang, C.-D. Xie, and H. Wang, Phys. Rev. Lett. 101, 073602 (2008).

[4] B.-W. Shiau, M.-C. Wu, C.-C. Lin, and Y.-C. Chen, Phys. Rev. Lett. 106, 193006 (2011).

[5] M. Fleischhauer, A. Imamoglu, and J. P. Marangos, Rev. Mod. Phys. 77, 633 (2005).

[6] S. E. Harris and L. V. Hau, Phys. Rev. Lett. 82, 4611 (1999).

[7] R. B. Li, L. Deng, and E. W. Hagley, Phys. Rev. Lett. 110, 113902 (2013).

[8] M. D. Lukin and A. Imamoglu, Phys. Rev. Lett. 84, 1419 (2000).

[9] S. Rebić, D. Vitali, C. Ottaviani, P. Tombesi, M. Artoni, F. Cataliotti, and R. Corbalán, Phys. Rev. A 70, 032317 (2004).

[10] D. Petrosyan and G. Kurizki, Phys. Rev. A 65, 033833 (2002).

[11] D. Petrosyan and Y. P. Malakyan, Phys. Rev. A 70, 023822 (2004).

[12] Z.-B. Wang, K.-P. Marzlin, and B. C. Sanders, Phys. Rev. Lett. 97, 063901 (2006).

[13] M. A. Antón, F. Carreño, O. G. Calderón, S. Melle, and I. Gonzalo, Opt. Commun. 281, 6040 (2008).

[14] C. Ottaviani, D. Vitali, M. Artoni, F. Cataliotti, and P. Tombesi, Phys. Rev. Lett. 90, 197902 (2003).

[15] C. Ottaviani, S. Rebic, D. Vitali, and P. Tombesi, Eur. Phys. J. D 40, 281 (2006).

[16] J. D. Pritchard, K. J. Weatherill, and C. S. Adams, Annual Review of Cold Atoms and Molecules (World Scientific, Singapore, 2013), Chap. 8, pp. 301-350.

[17] M. Saffman, T. G. Walker, and K. Mölmer, Rev. Mod. Phys. 82, 2313 (2010)

[18] K. Hammerer, A. S. Sorensen, and E. S. Polzik, Rev. Mod. Phys. 82, 1041 (2010).

[19] A. E. B. Nielsen and K. Molmer, Phys. Rev. A 81, 043822 (2010).

[20] D. Petrosyan and M. Fleischhauer, Phys. Rev. Lett. 100, 170501 (2008).

[21] J. D. Pritchard, D. Maxwell, A. Gauguet, K. J. Weatherill, M. P. A. Jones, and C. S. Adams, Phys. Rev. Lett. 105, 193603 (2010).

[22] T. Peyronel, O. Firstenberg, Q.-Y. Liang, S. Hofferberth, A. V. Gorshkov, T. Pohl, M. D. Lukin, and V. Vuletic, Nature (London) 488, 57 (2012).

[23] O. Firstenberg, T. Peyronel, Q.-Y. Liang, A. V. Gorshkov, M. D. Lukin, and V. Vuletic, Nature (London) 502, 71 (2013).

[24] A. V. Gorshkov, J. Otterbach, M. Fleischhauer, T. Pohl, and M. D. Lukin, Phys. Rev. Lett. 107, 133602 (2011).

[25] B. He, A. V. Sharypov, J.-T. Sheng, C. Simon, and M. Xiao, Phys. Rev. Lett. 112, 133606 (2014).

[26] D. Paredes-Barato and C. S. Adams, Phys. Rev. Lett. 112, 040501 (2014).
[27] M. Khazali, K. Heshami, and C. Simon, Phys. Rev. A 91, 030301(R) (2015).

[28] D. Yan, C.-L. Cui, Y.-M. Liu, L.-J. Song, and J.-H. Wu, Phys. Rev. A 87, 023827 (2013).

[29] Y.-M. Liu, X.-D. Tian, D. Yan, Y. Zhang, C.-L. Cui, and J.-H. Wu, Phys. Rev. A 91, 043802 (2015).

[30] D. Petrosyan, J. Otterbach, and M. Fleischhauer, Phys. Rev. Lett. 107, 213601 (2011).

[31] I. Friedler, D. Petrosyan, M. Fleischhauer, and G. Kurizki, Phys. Rev. A 72, 043803 (2005).

[32] S. Rebic, C. Ottaviani, G. Di Giuseppe, D. Vitali, and P. Tombesi, Phys. Rev. A 74, 032301 (2006).

[33] J. Gea-Banacloche, Phys. Rev. A 81, 043823 (2010).

[34] B. He and A. Scherer, Phys. Rev. A 85, 033814 (2012).

[35] Y.-M. Liu, D. Yan, X.-D. Tian, C.-L. Cui, and J.-H. Wu, Phys. Rev. A 89, 033839 (2014).

[36] A. Joshi and M. Xiao, Phys. Rev. A 72, 062319 (2005).

[37] M. D. Lukin, S. F. Yelin, M. Fleischhauer, and M. O. Scully, Phys. Rev. A 60, 3225 (1999).

[38] C.-L. Cui, J.-K. Jia, J.-W. Gao, Y. Xue, G. Wang, and J.-H. Wu, Phys. Rev. A 76, 033815 (2007).

[39] This also allows for a vanishing cross Kerr effect such that only Rydberg blockade is responsible for the conditional cooperative nonlinearity shown later.

[40] Each blockade radius can be interpreted as the smallest interatomic distance at which the dipole-dipole interaction moves the Rydberg level out of one EIT window from its center. See Refs. [22,30] for more details.

[41] K. Singer, J. Stanojevic, M. Weidemuller, and R. Cote, J. Phys. B: At. Mol. Opt. 38, S295 (2005)

[42] We find in Ref. [22] $N_{0}=2.0 \times 10^{12} \mathrm{~cm}^{-3}$ and $R_{b}=13.0 \mu \mathrm{m}$ have been achieved for rubidium atoms in experiment, which then yields $n_{\mathrm{SA}} \simeq 18350$.

[43] Both ground collective states $|G\rangle$ and $|M\rangle$ contain $n_{\mathrm{SA}} / 2$ atoms because each SA contains $n_{\mathrm{SA}}$ atoms and these atoms are equally distributed in ground atomic levels $|g\rangle$ and $|m\rangle$.

[44] V. Parigi, E. Bimbard, J. Stanojevic, A. J. Hilliard, F. Nogrette, R. Tualle-Brouri, A. Ourjoumtsev, and P. Grangier, Phys. Rev. Lett. 109, 233602 (2012).

[45] C. Ates, S. Sevincli, and T. Pohl, Phys. Rev. A 83, 041802(R) (2011).

[46] M. Tanasittikosol, J. D. Pritchard, D. Maxwell, A. Gauguet, K. J. Weatherill, R. M. Potvliege, and C. S. Adams, J. Phys. B: At. Mol. Opt. Phys. 44, 184020 (2011).

[47] A similar averaging has been used in Ref. [46] to describe the interaction effects in microwave dressed Rydberg atoms and in Ref. [44] to explain the large dispersive effects in an ensemble of cold Rydberg atoms. This approach has also been discussed in Refs. [30,35] and, in particular, in Ref. [45] where it has been supported by Monte Carlo simulations.

[48] A similar behavior can be observed around point $\beta$ and hence will not be reported here. 